\title{
Roles of Arabidopsis PSEUDO-RESPONSE REGULATOR (PRR) genes in the opposite controls of flowering time and organ elongation under long-day and continuous light conditions
}

\author{
Kanae Niinuma ${ }^{1}$, Norihito Nakamichi², Kana Miyata ${ }^{1}$, Takeshi Mizuno $^{3}$, \\ Hiroshi Kamada', Tsuyoshi Mizoguchi ${ }^{1, *}$ \\ ${ }^{1}$ Institute of Biological Sciences, University of Tsukuba, Tsukuba, Ibaraki 305-8572, Japan; ${ }^{2}$ Division of Biological \\ Science, Graduate School of Science, Nagoya University, Chikusa-ku, Nagoya 464-8602, Japan; ${ }^{3}$ Laboratory of \\ Molecular Microbiology, School of Agriculture, Nagoya University, Chikusa-ku, Nagoya 464-8601, Japan \\ *E-mail: mizoguchi@gene.tsukuba.ac.jpＴel: +81-298-53-6005 Fax: +81-298-53-7723
}

Received November 9, 2007; accepted November 26, 2007 (Edited by M. Sekine)

\begin{abstract}
The circadian clock regulates a wide variety of processes including the control of photoperiodic flowering and organ elongation in higher plants. Arabidopsis is a facultative long-day (LD) plant and flowers much earlier under LD and continuous light (LL) than short-day (SD) conditions. Although many of the genes required for the control of photoperiodic flowering have been identified, the precise mechanisms underlying the recognition of critical day or night lengths required for photoperiodic responses have not been fully clarified. To address this issue, we investigated circadian outputs in the lossof-function of PSEUDO-RESPONSE REGULATOR (PRR) genes, which are believed to be clock components, under LD and LL. Here we report that $p r r 9$ flowered earlier under LL but later under LD compared to wild-type plants, which showed an opposite control of flowering response under these conditions. Although flowering times under LD and LL were similar, prr9;prr7;prr5 mutant plants showed an opposite control of petiole elongation under LD and LL. Under LL, the prr9;prr7;prr5 mutant plants had shorter petioles but longer hypocotyls than those of wild-type plants. Based on our results, we propose some models to explain the organ-specific effect caused by mutations in Arabidopsis clock genes.
\end{abstract}

Key words: Circadian rhythm, photoperiodic flowering, PSEUDO-RESPONSE REGULATOR.

Circadian rhythms are oscillations in the biochemical, physiological, and behavioral functions of organisms that occur with approximate 24-h time periods with no external timing cues. This process enables an organism to phase its biological activities to the correct time of day. In higher plants, the circadian clock affects various processes, including the expression of many genes (Carpenter et al. 1994; Ernst et al. 1990; Staiger et al. 1999; Zhong et al. 1996), leaf movement (Engelmann et al. 1992), petal opening (Engelmann et al. 1978), and hypocotyl elongation (Dowson-Day and Millar 1999).

In the current model of the Arabidopsis oscillator, many circadian clock-associated genes have been identified through genetic studies. CIRCADIAN CLOCK ASSOCIATED 1 (CCA1), LATE ELONGATED HYPOCOTYL (LHY), and TIMING OF CAB EXPRESSION 1/PSEUDO-RESPONSE REGULATOR 1 (TOC1/PRRI) are believed to act as part of a central negative feedback loop (Alabadí et al. 2001). LHY and CCA1 encode Myb-related DNA-binding proteins and have partially redundant functions (Mizoguchi et al. 2002). Double loss-of-function of $L H Y$ and CCA1 (lhy;ccal) causes almost an arrhythmic expression of clock-controlled genes (Mizoguchi et al. 2002). LHY and CCA1 repress the expression of TOC1 through direct binding to the TOC1 promoter (Alabadí et al. 2001). In turn, $T O C 1$ feeds back either directly or indirectly to regulate CCAI and $L H Y$. In tocl mutants, circadian clock regulation still occurs, but its mutation has been shown to shorten the period in a variety of clockcontrolled processes (Somers et al. 1998). This indicates that TOC1 plays pivotal roles in various clock-controlled processes throughout development in Arabidopsis. Four PSEUDO-RESPONSE REGULATOR (PRR) genes, PRR9, $P R R 7, P R R 5$, and $P R R 3$, were identified as homologs of TOC1/PRR1 in the Arabidopsis genome (Matsushika et

Abbreviations: CCA1, CIRCADIAN CLOCK ASSOCIATED 1; CO, CONSTANS; ELF3, EARLY FLOWERING 3; FT, FLOWERING LOCUS T; GAI, GA INSENSITIVE; GI, GIGANTEA; Hd1, Heading date 1; Hd3a, Heading date 3a; LD, long-day; LHY, LATE ELONGATED HYPOCOTYL; LL, continuous light; PDPs, plant-derived pharmaceutical proteins; PRR, PSEUDO-RESPONSE REGULATOR; RT-PCR, reverse transcription-polymerase chain reaction; SD, short-day; TOC1, TIMING OF CAB EXPRESSION 1; TUB, TUBULIN; WT, wild-type.

This article can be found at http://www.jspcmb.jp/ 
al. 2000). Recent studies revealed that $P R R 9, P R R 7$, and PRR5 also play key roles in the control of circadian rhythms (Nakamichi et al. 2005a; Nakamichi et al. 2005b).

Determining the timing of flowering is critical for successful reproduction in plants, and many studies have been made on the photoperiodic flowering pathway (reviewed in Imaizumi and Kay 2006; Mizoguchi et al. 2007). In Arabidopsis, it was proposed that the precise control of the timing of $\mathrm{CO}$ expression, such that it is high during daytime only in long-day (LD) condition, is an essential factor for FLOWERING LOCUS T (FT) activation underlying the photoperiodic control of flowering (Roden et al. 2002; Suarez-Lopez et al. 2001; Yanovsky and Kay 2002).

Arabidopsis is a facultative LD plant and flowers much earlier under LD compared to short-day (SD) conditions. Comparative analysis of Arabidopsis and the SD plant rice demonstrated that functional differences between the Arabidopsis $\mathrm{CO}$ and its rice ortholog, Heading datel (Hdl) are the basis of the reversal in the photoperiodic response type (Hayama and Coupland 2004). In rice, $H d l$ suppresses flowering under LD conditions by repressing expression of the rice ortholog of FT, Heading date3a (Hd3a), whereas in Arabidopsis, $\mathrm{CO}$ induces flowering by activating $F T$ expression (Hayama and Coupland 2004). FT and Hd3a are candidates of a floral hormone, florigen (Corbesier et al. 2007; Tamaki et al. 2007). We recently found that lhy;ccal double mutant plants exhibited unique phenotypes under different photoperiodic conditions (Fujiwara et al. unpublished data). Although lhy; ccal mutation accelerates flowering of Arabidopsis under light/dark cycles such as LD and SD, lhy;ccal mutants grown under continuous light (LL) flowered later than under SD conditions, suggesting that $L H Y$ and $C C A 1$ play important roles in the photoperiodic response of Arabidopsis.

Both floral activators and repressors play key roles in the control of flowering (Hartmann et al. 2000; Lee et al 2000; Michaels and Amashino 1999; Putterill et al. 1995). A balance between these activities may be key for either accelerating or delaying flowering. However, a precise molecular mechanism underlying the control of the phase transition from vegetative to reproductive growth has not been elucidated. Under different photoperiods, plants show different shapes. The difference in shapes depends largely on controlling the elongation of organs such as hypocotyls, petioles, and stems. The circadian clock is reported to affect not only flowering but also elongation of plant organs. We can easily speculate that lengths of plant organs may also be controlled by a balance between two opposite activities, lengthening and shortening, as in the case of flowering. Reversal of flowering response types of the lhy;ccal in the light/dark cycles (LD and SD) and LL suggests that $L H Y$ and CCAI might have distinct roles under the different photoperiodic conditions. We believe that revealing the possible hidden roles of clock proteins will be helpful to clarify the mechanism underlying the control of phase transitions.

In this study, mutations of $P R R$ genes were tested for their effects on flowering and organ elongation under LD and LL. Loss-of-function of PRR9 slightly accelerated flowering under LL but delayed it under LD conditions, showing another example of reversed flowering responses between LD and LL. In contrast, prr9;prr7;prr5 mutant plants delayed flowering under both LD conditions and LL. Although the prr9;prr7;prr 5 triple mutant plants did not show the reversal of flowering response type, an opposite control of petiole elongation was observed in the prr9;prr7;prr5 under LD and LL. Based on our results, we propose some models to explain the organ-specific effect caused by mutations of Arabidopsis clock genes.

\section{Materials and methods}

Plant material, growth conditions, and analysis of leaf and hypocotyl phenotypes of prr mutants

Arabidopsis thaliana accession Columbia (Col) plants were used as the wild type (WT). Mutants prr9-10/prr7-11/prr5-11 (prr9;prr7;prr5), prr9-10/prr7-11 (prr9;prr7), prr9-10/prr5-11 (prr9;prr5), prr7-11/prr5-11 (prr7;prr5), prr9 (SALK-007511), prr7 (SALK-030430), and prr5 (KAZUSA-KG24599) have been previously described (Nakamichi et al. 2005a, Nakamichi et al. 2005b). Seeds were imbibed and cold treated at $4{ }^{\circ} \mathrm{C}$ for 3 days in the dark before germination under light. Plants were grown in controlled environment rooms at $22^{\circ} \mathrm{C}$. Light conditions were either LD ( $16 \mathrm{~h}$ light $/ 8 \mathrm{~h}$ dark) or LL (continuous white light) with a photon flux density of about $40 \mu \mathrm{mol} \mathrm{m}^{-2} \mathrm{~s}^{-1}$.

Hypocotyl length was measured in 14-day-old plants. Leaf blades and petioles of the 3rd and 5th leaves were measured at 3-weeks and 30 days, respectively, after sowing.

\section{Measurement of flowering time}

Plants were grown as described above. Flowering time was scored by growing plants on soil under LD and LL and counting the number of rosette and cauline leaves on the main stem after bolting. Data are presented as the means \pm SE ( $n \geqq 11$ ). Measurement of flowering time was performed at least twice, with similar results.

\section{Preparation of RNA and Semiquantitative RT- PCR}

Plants were sown as described above and grown on soil for 10 days. Aerial parts were used for RNA preparation. RT-PCR was performed with $1 \mu \mathrm{g}$ of total RNA using a SuperScript FirstStrand Synthesis System for RT-PCR (Invitrogen, Carlsbad, CA, USA). cDNA was diluted to $100 \mu$ l with TE buffer, and $1 \mu 1$ of diluted cDNA was used for PCR amplification by TaKaRa Extaq (TaKaRa, Shiga, Japan). For RT-PCR expression, the following primers were used: $\mathrm{CO}, 5^{\prime}$-ACGCCATCAGCGAGTTCC-3' and 5'-AAATGTATGCGTTATGGTTAATGG-3' (Suarez-Lopez et al. 2001); $F T, 5^{\prime}$-ACAACTGGAACAACCTTTGGCAATG-3' and 5'-ACTATATAGGCATCATCACCGTT- 
CGTTACTCG-3' (Bláquez and Weigel 1999); TUBULIN 2 $(T U B), 5^{\prime}$-CACCATGGAAGAAGTGAAGACG-3' and 5'-GACTGTCTCCAAGGGTTCCAG-3'. Numbers of PCR cycles were as follows: 25 cycles for $C O, 28$ cycles for $F T$, and 21 cycles for $T U B$. Annealing temperatures were $60^{\circ} \mathrm{C}$ for $C O$ and $58^{\circ} \mathrm{C}$ for $F T$ and $T U B$. The PCR products were separated on $1.5 \%$ agarose gels and transferred to Biodyne B membranes (Nippon Genetics, Tokyo, Japan). The membranes were hybridized with radioactive probe DNAs in a hybridization solution that contained $5 \times \mathrm{SSC}(1 \times \mathrm{SSC}=0.15 \mathrm{M} \mathrm{NaCl}$ and $0.015 \mathrm{M}$ sodium citrate), $0.1 \%$ SDS, $0.1 \%$ sarkosyl, $0.75 \%$ Blocking reagent (Boehringer Mannheim, Mannheim, Germany), and $5 \%$ dextran sulfate sodium salt at $65^{\circ} \mathrm{C}$ for $16 \mathrm{~h}$. The blot was washed with $2 \times$ SSC and $0.1 \%$ SDS for $20 \mathrm{~min}$, and then $0.5 \times \mathrm{SSC}$ and $0.1 \%$ SDS for $10 \mathrm{~min}$ at $65^{\circ} \mathrm{C}$, after which the hybridization signal was visualized using the BioImaging Analyzer (BAS 5000; Fuji Photo Film, Tokyo, Japan); signal intensity was quantified with Science Lab 98 Image Gauge software (version 3.1; Fuji Photo Film). Values reported are means from data at circadian time $0,4,8,12,16,20,24 \mathrm{~h}$ of 10-day-old plants. Results were normalized with respect to TUB levels. RT-PCR analyses were performed at least twice and usually with independent RNA samples. Similar results were obtained from two experiments.

\section{Results}

\section{prr9 flowered slightly earlier under LL but later under LD conditions than the wild type}

To test whether clock mutants with arrhythmic phenotype displayed the reversal of flowering response between $\mathrm{LD}$ and LL, the flowering time of the prr9;prr7;prr5, together with prr double and single mutants, were scored under both LD and LL (Figure 1). Unlike in the case of lhy;ccal, prr9;prr7;prr5, mutant plants flowered later than the WT (Col) under both LD (Nakamichi et al. 2005a; Figure 1A, left, Table 1) and LL (Figure 1A, right, Table 1). Table 1 summarizes the results obtained in this study (Table 1, in bold upper case letters). The flowering phenotypes of prr mutants under LD and SD that have been reported (Table 1, not bold) are also shown. In Table 1, "S." and "E." denote "slightly" and "extremely," respectively. This result suggests that the arrhythmic phenotype did not explain the reversal of flowering phenotype observed in lhy;ccal mutants.

Under LD conditions, prr9, prr7, prr5, and prr9;prr5 flowered slightly later than WT (Col) plants, as previously reported (Nakamichi et al. 2005a; Figure 1A, left, Table 1). The late flowering phenotypes of prr9;prr7, prr7;prr5, and prr9;prr7;prr5 were more greatly pronounced under LD conditions. Under LL, similar results were obtained to those under LD conditions, except for prr9;prr7 and prr9 (Figure 1A, right, Table 1). Under LD conditions, the prr9;prr7 mutant plants flowered with over 65 leaves more than the WT (Col) control. In contrast, under LL, the prr9;prr7 mutant plants flowered with only 30 fewer leaves and a
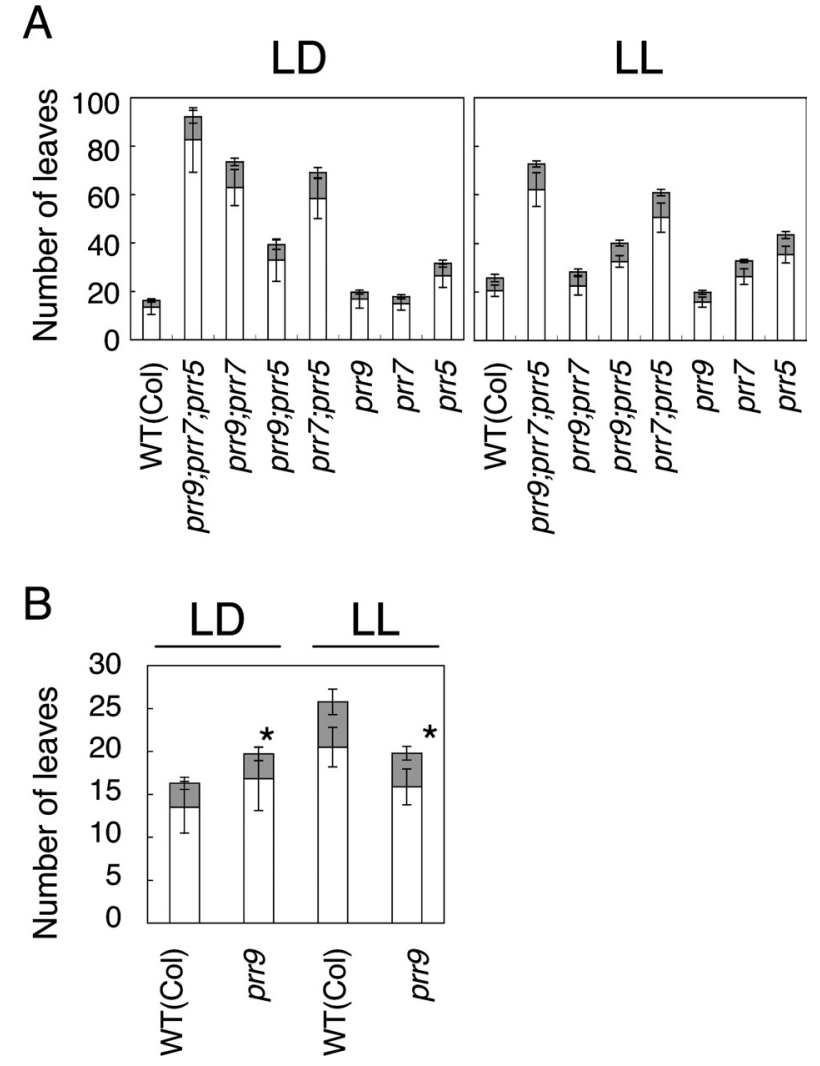

Figure 1. The flowering times in prr mutants under LD and LL. (A) The flowering time in WT (Col), prr9, prr 7, prr5, prr9;prr 7, prr9;prr5, prr7;prr5, and prr9;prr7;prr5 was measured under LD (left) or LL conditions (right). Flowering time was scored by counting the number of rosette (bottom box) and cauline (top box) leaves on the main stem. Error bars represent SE $(n \geqq 10)$. Each experiment was performed at least twice, with similar results. (B) Comparison of the flowering time in the WT (Col) and prr9 between LD and LL conditions. Asterisks denote statistical significance in comparison to values of the WT (Col) (Student's t-test, $P<0.05$ ).

similar number to that of the WT (Col). Additionally, prr $9 ; \operatorname{prr} 7$ mutants produced more leaves than the prr9 mutant control but fewer leaves than the prr7 mutant control. The prr9 flowered slightly later under LD condition but slightly earlier under LL (Figure 1B, Table1). The flowering phenotype of prr9 mutants under SD has not been determined yet (Table 1; ND indicates "not determined").

\section{CO expression levels did not explain FT expression levels and flowering times of prr mutants under $L L$}

The expression of $C O$ shows a circadian rhythm with the peak at around $12 \mathrm{~h}$ after dawn (Suarez-Lopez et al. 2001). The "coincidence model" proposes that LD can trigger flowering because the expression of $\mathrm{CO}$ coincides with the exposure of plants to light (Roden et al. 2002; Suarez-Lopez et al. 2001; Yanovsky and Kay 2002). Therefore, in WT (Col) plants grown under LD conditions, the $C O$ mRNA accumulates at dusk and under light 
Table 1. Summary view of the prr mutants under LD and LL

\begin{tabular}{|c|c|c|c|c|c|c|c|c|c|c|}
\hline & \multirow{2}{*}{\multicolumn{2}{|c|}{ Hypocotyl }} & \multicolumn{4}{|c|}{ Leaf } & \multirow{2}{*}{\multicolumn{3}{|c|}{ Flowering }} & \multirow{3}{*}{ References } \\
\hline & & & \multicolumn{2}{|c|}{ Blade } & \multirow{2}{*}{$\begin{array}{c}\text { Petiole } \\
\text { LL }\end{array}$} & \multirow{2}{*}{$\frac{\text { Petiole }}{\text { LD }}$} & & & & \\
\hline & LL & LD & LL & LD & & & LL & LD & SD & \\
\hline prr9;prr7;prr5 & Long & E. Long & Short & \pm & Short & Long & E. Late & E. Late & Late & $\begin{array}{l}\text { Nakamichi et al. (2007); } \\
\text { Nakamichi et al. (2005a) }\end{array}$ \\
\hline prr9;prr7 & Long & Long & \pm & \pm & S. Long & Long & \pm & Late & Late & $\begin{array}{l}\text { Nakamichi et al. (2007); } \\
\text { Nakamichi et al. (2005a) }\end{array}$ \\
\hline prr9;prr5 & Long & S. Long & \pm & \pm & Long & \pm & Late & $\begin{array}{l}\text { Late } \\
\text { (S. Late) }\end{array}$ & Late & $\begin{array}{l}\text { Nakamichi et al. (2007); } \\
\text { Nakamichi et al. (2005a) }\end{array}$ \\
\hline prr7;prr5 & E. Long & E. Long & $\begin{array}{c} \pm \\
\text { (S. Short) }\end{array}$ & \pm & S. Long & S. Long & E. Late & Late & Early & $\begin{array}{l}\text { Nakamichi et al. (2007); } \\
\text { Nakamichi et al. (2005b) }\end{array}$ \\
\hline prr9 & S. Long & S. Long & \pm & $\begin{array}{c} \pm \\
\text { (S. Long) }\end{array}$ & \pm & \pm & S. Early & S. Late & ND & Nakamichi et al. (2005a) \\
\hline prr7 & S. Long & Long & $\begin{array}{c} \pm \\
\pm \\
\text { (S. Long) }\end{array}$ & $\begin{array}{c} \pm \\
\text { (S. Long) }\end{array}$ & Long & S. Long & S. Late & $\begin{array}{l}\text { S. Late } \\
\text { (Late) }\end{array}$ & Early & $\begin{array}{l}\text { Nakamichi et al. (2007); } \\
\text { Yamamoto et al. (2003) }\end{array}$ \\
\hline prr 5 & S. Long & S. Long & \pm & \pm & S. Long & \pm & Late & $\begin{array}{l}\text { Late } \\
\text { (S. Late) }\end{array}$ & Early & $\begin{array}{l}\text { Nakamichi et al. (2007); } \\
\text { Yamamoto et al. (2003) }\end{array}$ \\
\hline
\end{tabular}

The results in bold upper case were obtained in this study. "S." and "E." denote "slightly" and "extremely", respectively. ND indicates "not determined".

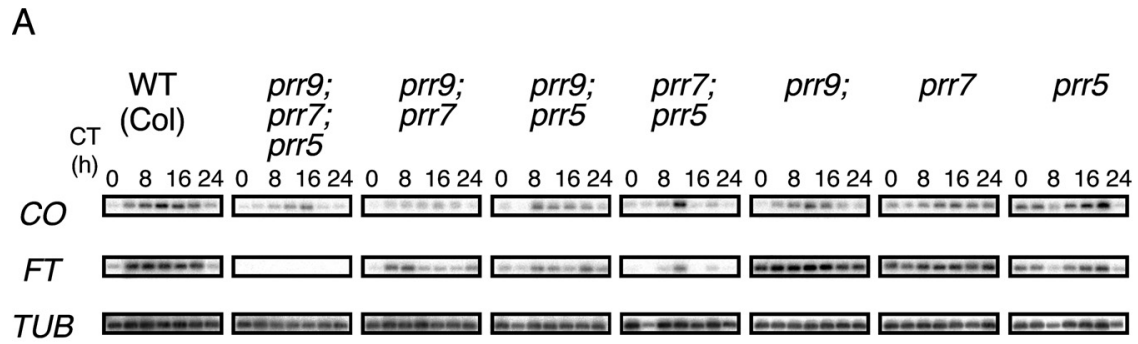

B

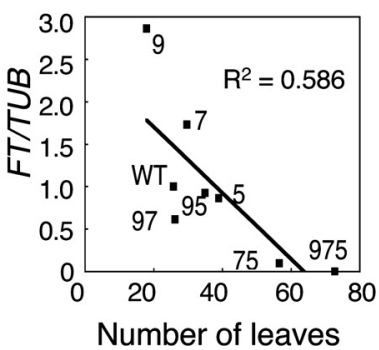

C

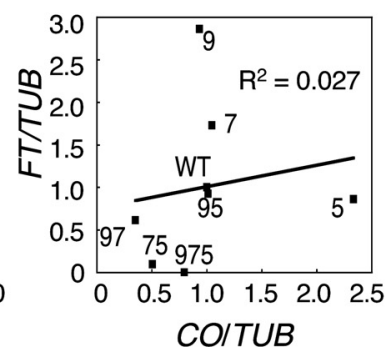

$\mathrm{D}$

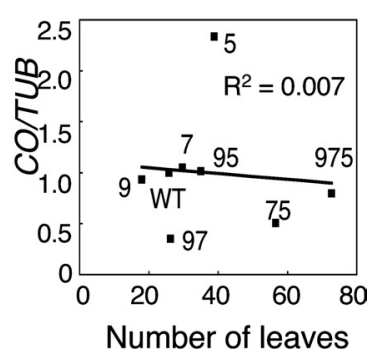

Figure 2. The expression of photoperiodic flowering-related genes and correlation with flowering time in prr mutants under LL. (A) The expression of $C O, F T$, and TUB genes was analyzed by RT-PCR in the WT (Col), prr9, prr7, prr5, prr9;prr7, prr9;prr5, prr7;prr5, and prr 9; prr7; prr 5 grown under LL. RNA was extracted at 4-h intervals for $24 \mathrm{~h}$ from the plants. Each experiment was performed at twice, with similar results. (B-D) Correlation of (B) FT expression-flowering time. (C) FT-CO expression and (D) CO expression-flowering time in prr mutants under LL. Each $F T$ and $C O$ expression indicates the mean value of seven samples (at $0,4,8,12,16,20$, and $24 \mathrm{~h}$ ), normalized with respect to $T U B$ expression.

(Suarez-Lopez et al. 2001).

We measured the mRNA levels of $C O$ and $F T$ in prr mutants under LL (Figure 2A) to examine whether these flowering phenotypes (Figure 1A) were mediated through the CO-FT flowering pathway under LL. RNA was extracted at 4-h intervals for $24 \mathrm{~h}$ from plants growing under LL. The $F T$ mRNA level showed a strong correlation with the flowering times of these mutants (Figure $2 \mathrm{~B} ; \mathrm{R}^{2}=0.586$ ). Early flowering plants including the WT (Col), prr9, and prr7 plants accumulated more $F T$ mRNA, whereas late flowering plants such as prr 9;prr7;prr5 and prr7;prr5 accumulated less. However, correlations between the mRNA levels of FT and $C O$ (Figure $2 \mathrm{C} ; \mathrm{R}^{2}=0.027$ ) and between $C O$ expression and flowering times (Figure 2D; $\mathrm{R}^{2}=0.007$ ) under LL were not significant. Although $F T$ expression was below detectable levels in prr9;prr7;prr5 under LL, $C O$ mRNA abundance had decreased to only half the level of WT (Col) plants. This result suggests that the prr9;prr7;prr 5 was more sensitive to the decrease in $C O$ mRNA level, or that a different pathway might play a role in the downregulation of $F T$ expression. Although the $C O$ expression 
of prr $9 ; \operatorname{prr} 7$ decreased to approximately $70 \%$ of WT (Col) plants, the flowering time and FT mRNA level of the double mutant were similar to those of WT (Col) plants under LL. These results suggest that prr9;prr7;prr5 and prr9;prr7 slightly decreased the $C O$ mRNA level, but this did not explain the downregulation of $F T$ expression.

\section{prr9 partially suppressed the long hypocotyl phenotype of prr7;prr5 under both LD and LL}

The elongation rates of organs such as hypocotyls oscillate with a circadian rhythm and are controlled by a circadian clock (Dowson-Day and Millar 1999). Several mutants lacking clock-associated genes have altered organ lengths in Arabidopsis (Nozue and Maloof 2006; Schaffer et al. 1998; Somers et al. 2000; Wang and Tobin 1998). However, the mechanisms underlying the clockcontrolled regulation of organ elongation are not yet clear. Some clock-associated genes are implicated in certain light signal transduction pathways (Quail 2002). The inhibition of hypocotyl elongation under light within a given spectrum was analyzed to investigate their role in each light signal transduction. The prr mutants showed a wide variety of hypocotyl lengths when these mutants were grown under continuous red light (Nakamichi et al. 2005a). Among them, loss-of-function of PRR7 displayed hypo-sensitivity to red light and a synergistic effect with prr5.

Under LD and LL with white light, prr7 showed long hypocotyls and a synergistic effect with prr5 (Figure 3A, Table 1). Under both LD and LL, the hypocotyl length of prr7;prr 5 was longest. Although prr9 mutant plants had slightly longer hypocotyls than those of the WT $(\mathrm{Col})$ both under LD and LL, prr9 partially suppressed the hypocotyl elongation of prr7; prr5 (Figure 3A).

\section{prr9;prr7;prr5 lengthened the petiole length under LD conditions but shortened it under LL}

In many species of dicotyledonous plants, the leaf mainly consists of a blade and a petiole (Denglar and Tsukaya 2001). The leaf blade is a wide and flat organ specialized for effective photosynthesis. The leaf petiole supports the leaf blade and orients it to positions that are more appropriate for photosynthesis. To analyze the regulation of organ elongation by $P R R$ genes, we measured the lengths of leaf blades and petioles under LD and LL (Figure 4A, B, Table 1). Leaf blades and petioles were analyzed in 3rd leaves (Figure 4A) and 5th (Figure 4B) leaves at the ages of approximately 3 -weeks and 30 days, respectively. Under LL, the lengths of the leaf blades of all prr mutants except prr9;prr7;prr5 were similar to those under LD conditions. The lengths of both the leaf blade and petiole were shortened in prr9;prr7;prr5 under LL (Figure 4A, B, C, Table1). Interestingly, however, the prr9;prr7;prr5

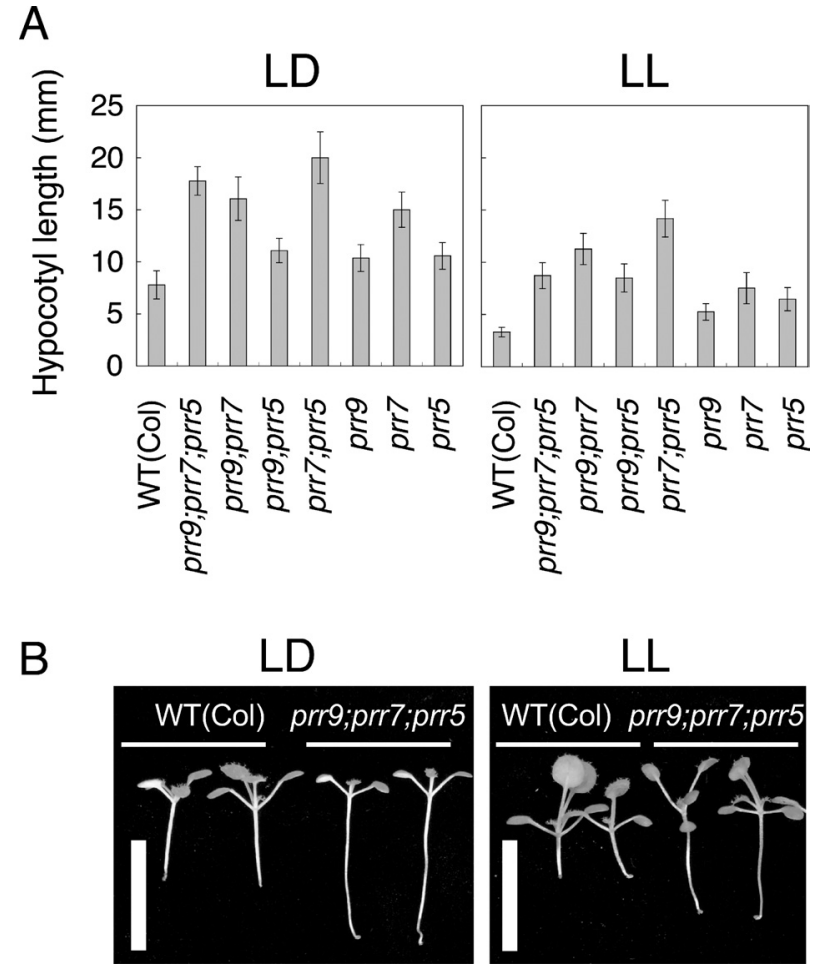

Figure 3. Hypocotyl length of prr mutants under LD and LL. (A) Hypocotyl length of the WT (Col), prr9, prr7, prr5, prr9;prr7, prr9;prr5, prr7;prr5, and prr9;prr7;prr5 grown under LL. Seedlings were grown for 14 days under LD (left) and LL conditions (right). Error bars represent the $\mathrm{SE}(\mathrm{n} \geqq 10)$. Each experiment was performed at least twice, with similar results. (B) Seedlings of WT (Col) plants (the left two seedlings in each panel) and prr9;prr7;prr5 plants (the right two seedlings in each panel) under LD (left panel) and LL (right panel). Plants at 14 days after sowing. Scale bar $=10 \mathrm{~mm}$.

mutant exhibited longer petioles than those of the WT (Col) under LD conditions (Figure 4A, B, C, Table 1). prr9;prr7;prr 5 had approximately $73 \%$ shorter petioles and $93 \%$ longer hypocotyls compared to the WT (Col) under LL (Figure 4D). In contrast, the prr9;prr7;prr5 plants had 38\% longer petioles and 79\% longer hypocotyls than those of the WT (Col) under LD conditions (Figure 4D). Therefore, we observed an opposite control of organ elongation between the petiole and hypocotyl only in prr9;prr7;prr5 grown under LL.

Next, we compared cell lengths of these organs (Figure 4E). The hypocotyl is usually composed of approximately 22 cells at the longitudinal axis and its elongation depends on cell elongation (Gendreau et al. 1997). In contrast, petiole elongation is associated with both cell division in the shoot apical meristem and cell elongation (Tsukaya et al. 2002). Petioles of the prr9;prr7;prr5 mutant plants had shorter cell length than the WT (Col) under LL (Figure 4E). In contrast, hypocotyls of prr9;prr7;prr 5 had longer cells than the WT (Col) under LL. These results suggest that this opposite control of organ elongation between the petioles and hypocotyls of prr9;prr7;prr5 under LL were based 


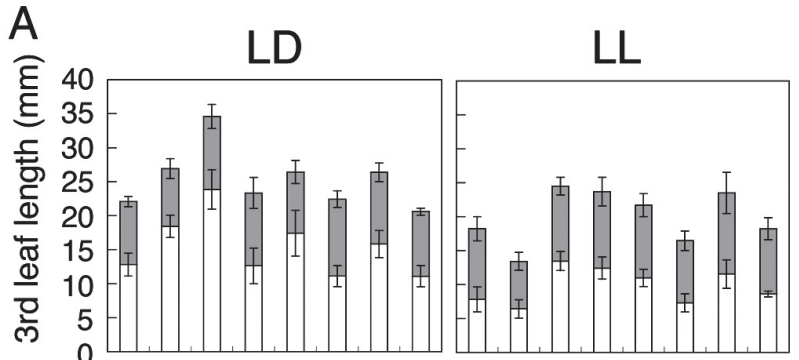

$\mathrm{B}$
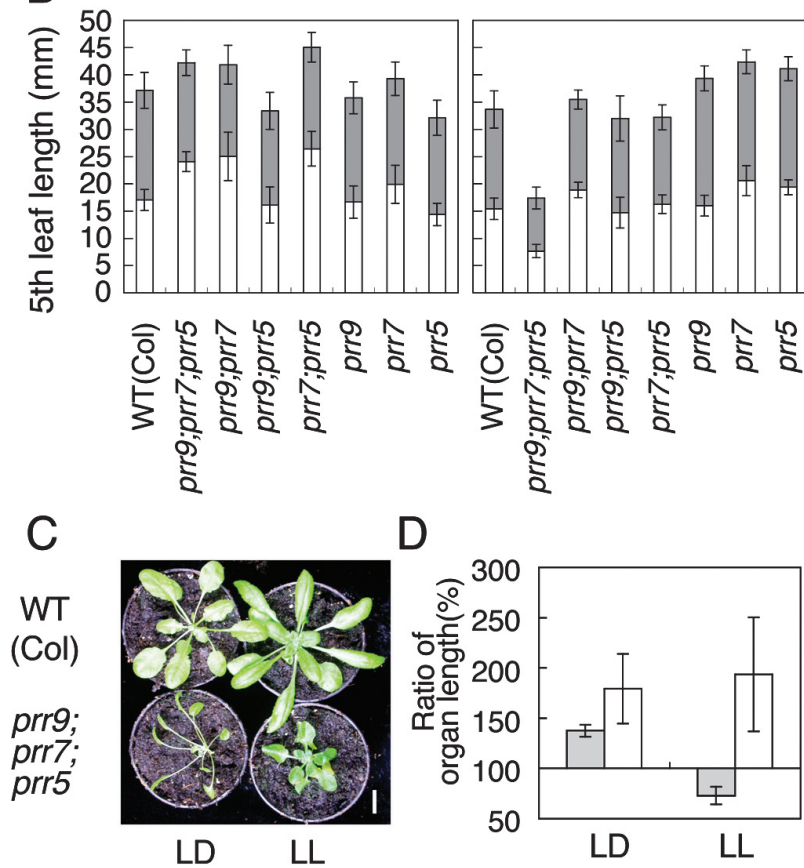

$E$

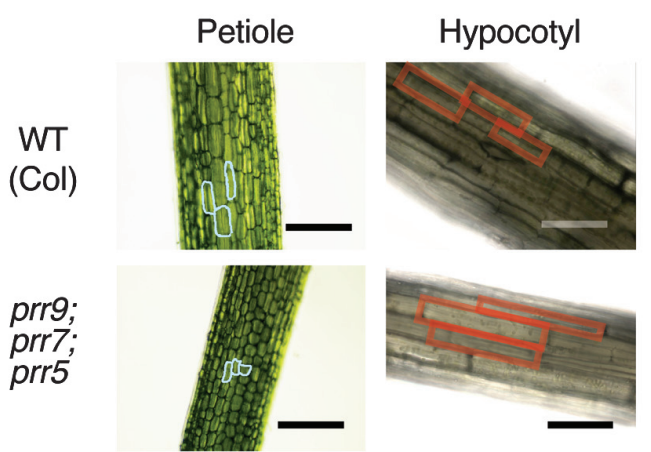

Figure 4. Leaf length phenotypes of prr mutants under LD and LL. (A,B) Mean of the leaf blade (closed boxes) and petiole (open boxes) length in the WT (Col), prr9, prr7, prr5, prr9;prr7, prr9;prr5, prr7;prr5, and prr9;prr7;prr5 grown under LD (left) and LL conditions (right). The $3 \mathrm{rd}$ (A) and 5th (B) leaves were measured 3 weeks and 30 days, respectively, after sowing. Error bars represent the $\mathrm{SE}(\mathrm{n} \geqq 10)$. Each experiment was performed at least twice, with similar results. (C) Leaf phenotype of the WT (Col; upper) and prr9;prr7;prr5 mutants (lower). Plants were grown for 30 days under LD (left) and LL (right) at $22^{\circ} \mathrm{C}$. Scale bar $=10 \mathrm{~mm}$. (D) Percent petiole (closed box) and hypocotyl (open box) lengths of prr9;prr7;prr 5 relative to the WT (Col). Values are based on triplicate experiments $(n \geqq 10)$. Error bars represent the SE. (E) Microscopic images of WT (Col) plants (upper) and $\operatorname{prr}$ 9;prr7;prr5 mutants (lower) under LL. 3rd leaves of the petiole (left sides) at 3 weeks old and hypocotyls (right sides) at 14 days old. Scale bar $=0.3 \mathrm{~mm}$. on the lengths of the cells.

\section{Discussion}

Numerous studies have been conducted on the mechanism of photoperiodism and the circadian clock, and recent reports have shed new light on the photoperiodic flowering in particular (Kim et al. 2007; Sawa et al. 2007). In this paper, we focused on characterizing the reversal of phenotype (e.g., flowering time and organ elongation) between different photoperiodic conditions such as LD and LL. Arabidopsis WT (Col) plants flower much earlier under LD and LL than SD conditions. However, if mutation of a certain gene showed a reversal of phenotype between LD and LL, the gene probably plays an important role in the maintenance of photoperiodicity. In addition, little attention has been given to LL in the study of photoperiodicity, and an analysis of phenotype may reveal the mechanism of photoperiodism. For these reasons, we investigated the phenotype of flowering and petiole and hypocotyl lengths in single, double, and triple mutants of PRR9, PRR7, and PRR5 grown under LD and LL.

We investigated the flowering phenotype of prr mutants. We focused more on prr9;prr7;prr 5 than other mutants to test whether an arrhythmic phenotype was associated with a reversal of flowering response types between LD and LL, e.g., in the case of lhy;ccal. prr9;prr7;prr 5 showed extremely late flowering under LD conditions (Nakamichi et al. 2005a; Figure 1A, left, Table 1) and LL (Figure 1A, right, Table 1), indicating that a severe arrhythmic phenotype was not always associated with the reversal of flowering response between LD and LL. Although the effects were rather small, prr9 mutant plants did show a reversal of flowering response types under LD and LL (Figure 1A, B). Interestingly, $p r r 9 ; p r r 7$ showed early flowering with a similar number of leaves as the WT (Col) under LL and flowered much later than the WT (Col) under LD conditions (Figure 1A). This indicates that the PRR9, $P R R 7$, and $P R R 5$ genes accelerate flowering under LD conditions, but $P R R 5$ appears to be especially important for the acceleration of flowering under LL. The prr7; prr 5 mutants flowered later than the WT (Col) plants under LD conditions, and did so only after developing a similar number of leaves as the WT $(\mathrm{Col})$ under SD conditions (Nakamichi et al. 2005a). These results suggest that PRR9, PRR7, and PRR5 in WT (Col) plants may have distinct roles in the acceleration of flowering under different photoperiodic conditions, such as LL, LD, and SD.

We analyzed the expression levels of photoperiodic flowering related genes, $C O$ and $F T$, under LL (Figure 2A). The external coincidence model of photoperiodic flowering proposes that $\mathrm{CO}$ induces expression of the 
FT under light condition (Roden et al. 2002; SuarezLopez et al. 2001; Yanovsky and Kay 2002). Under LD conditions, PRR9, PRR7, and PRR5 have been shown to control the mRNA levels of $C O$ and $F T$ and regulate flowering (Nakamichi et al. 2007). It has been proposed that reduced expression of $C O$ at Zeitgeber time $12 \mathrm{~h}$ may be responsible for the late flowering phenotype in prr9;prr7 under LD conditions (Nakamichi et al. 2007). prr9;prr7 mutants under LL showed reduced $C O$ expression but the expression level of $F T$ was similar to that of WT (Col) plants (Figure 2A). In addition, prr9;prr7;prr5 mutants showed a similar level of $C O$ expression to those of WT (Col) plants, suggesting a pathway that regulates $F T$ expression independent of $C O$ function. The characterization of $\operatorname{prr} 9 ; \operatorname{prr} 7 ; \operatorname{prr} 5 ; \mathrm{co}$ mutant plants will be helpful in testing this possibility.

The elongation rate of hypocotyls has been reported to oscillate with a circadian rhythm (Dowson-Day and Millar 1999). The elongation of petioles is also suggested to be controlled by the circadian clock because mutants with some clock components alter petiole lengths (Daniel et al. 2004) and the circadian rhythm of leaf angle is driven by petiole elongation (Engelmann and Johnsson 1998). Regulating the lengths of organs such as petioles and hypocotyls is critical for plants just after germination to perform efficient photosynthesis and to survive in their natural environments. However, mechanisms underlying the clock-controlled regulation of organ elongation have remained unclear. For example, how each clock protein contributes to the regulation and whether common or distinct pathway(s) regulate the lengths of petioles/ hypocotyls have not yet been determined. This is partly because all of the Arabidopsis mutants with altered lengths of petioles and hypocotyls reported so far show either lengthening or shortening of both organs. For example, mutants with altered sensitivity to light and hormones have been identified that exhibit either long (hy3 and early flowering 3 (elf3)) or short (ga insensitive (gai) and lhy;ccal) petioles/hypocotyls under LD conditions (Kim et al. 2005; Mizoguchi et al. 2005). These reports suggest that a common pathway may control the lengths of two distinct organs of plants.

In this study, we observed a similar tendency in the elongation of hypocotyls and petioles in most of the prr mutants under LL (Figures 3A, 4A, B), consistent with the well established concept described above. However, we found an apparently opposite phenotype between the petiole and hypocotyl in prr9;prr7;prr5 when grown under LL (Figures 3A, B, 4A, B, C, D). To elucidate a mechanism underlying the opposite control of organ elongation between petioles and hypocotyls, we measured the cell lengths of these two organs. Under LL, prr9;prr7;prr5 mutants had shorter cell lengths in petioles but longer lengths in hypocotyls than those of WT (Col) plants (Figure 4E). These results suggest that the opposite phenotype of organ length between these two organs in prr9;prr7;prr5 may depend on the difference of cell lengths in these two organs, and that the circadian clock may have organ-specific mechanisms to control cell elongation.

Based on our results, we propose two models to explain the mechanisms with which the circadian clock controls elongation of two different organs. "Model A" predicts the existence of organ-specific circadian clocks that regulate similar pathways involved in cell elongation in two organs. One of the loss-of-function alleles of GIGANTEA (GI), gi-2, shortened the leaf movement period but caused a gradual lengthening of the luminescence and RNA transcript abundance rhythms (Park et al. 1999), suggesting that independent circadian oscillators might separately control different outputs. "Model B" suggests that organ-specific pathways controlling cell elongation under a circadian clock may result in differential regulation of cell elongation between petioles and hypocotyls. Alternatively, a combination of "Model A" and "Model B" may also explain the mechanisms with which the circadian clock controls the elongation of two different organs.

\section{Acknowledgments}

We thank our colleagues for their support and helpful comments during our work, especially Dr. Riichiro Yoshida and Dr. Atsushi Oda for their valuable advices.

\section{References}

Alabadí D, Oyama T, Yanovsky MJ, Harmon FG, Mas P, Kay SA (2001) Reciprocal regulation between TOC1 and LHY/CCA1 within the Arabidopsis circadian clock. Science 293: 880-883

Bláuez MA, Weigel D (1999) Independent regulation of flowering by phytochrome B gibberellins in Arabidopsis. Plant Physiol 120: $1025-1032$

Carpenter CD, Kreps JA, Simon AE (1994) Genes encoding glycine-rich Arabidopsis thaliana proteins with RNA-binding motifs are influenced by cold treatment an endogenous circadian rhythm. Plant Physiol 104: 1015-1025

Corbesier L, Vincent C, Jang S, Fornara F, Fan Q, Searle I, Giakountis A, Farrona S, Gissot L, Turnbull C, Coupland G (2007) FT protein movement contributes to long-distance signaling in floral induction of Arabidopsis. Science 316: $1030-1033$

Daniel X, Sugano S, Tobin EM (2004) CK2 phosphorylation of CCA1 is necessary for its circadian oscillator function in Arabidopsis. Proc Natl Acad Sci 101: 3292-3297

Dengler NG, Tsukaya H (2002) Leaf morphogenesis in dicotyledons: current issues. Int J Plant Sci 162: 729-745

Dowson-Day MJ, Millar, AJ (1999) Circadian dysfunction causes aberrant hypocotyls elongation patterns in Arabidopsis. Plant $J$ 17: $63-71$

Engelmann W, Johnsson A (1998) Rhythms in organ movement. In: Lumsden PJ, Millar AJ (eds) Biological Rhythms Photoperiodism in Plants. BIOS Scientific, Oxford, pp 35-50 
Engelmann W, Johnsson A, Karlsson HG (1978) Attenuation of the petal movement rhythm in Kalanchoe with light pulses. Physiol Plant 43: 68-76

Engelmann W, Karl S, Phen CJ (1992) Leaf movement rhythm in Arabidopsis thaliana. Z Naturforsch C J Biosci 47: 925-928

Ernst D, Apfelbock A, Bergmann A, Weyrauch C (1990) Rhythmic regulation of the light-harvesting chlorophyll $\mathrm{a} / \mathrm{b}$ protein the small subunit of ribulose-1,5-bisphosphate carboxylase mRNA in rye seedlings. Photochem Photobiol 52: 29-33

Gendreau E, Traas J, Desnos T, Grandjean O, Caboche M, Hofte H (1997) Cellular basis of hypocotyl growth in Arabidopsis thaliana. Plant Physiol 114: 295-305

Hartmann U, Hohmann S, Nettesheim K, Wisman E, Saedler H, Huijser P (2000) Molecular cloning of SVP: A negative regulator of the floral transition in Arabidopsis. Plant $J$ 21: 351-360

Hayama R, Coupland G (2004) The molecular basis of diversity in the photoperiodic flowering responses of Arabidopsis and rice. Plant Physiol 135: 677-684

Imaizumi T, Kay SA (2006) Photoperiodic control of flowering: not only by coincidence. Trends Plant Sci 11: 550-558

Kim GT, Yano S, Kozuka T, Tsukaya H (2005) Photomorphogenesis of leaves: Shade-avoidance syndrome differentiation of sun/shade leaves. Photochem Photobiol Sci 4: 770-774

Kim WY, Fujiwara S, Suh SS, Kim J, Kim Y, Han L, David K, Putterill J, Nam HG, Somers DE (2007) ZEITLUPE is a circadian photoreceptor stabilized by GIGANTEA in blue light. Nature 20: $356-360$

Lee H, Suh S-S, Park E, Cho E, Ahn JH, Kim S-G, Lee JS, Kwon YM, Lee I (2000) The AGAMOUS-LIKE 20 MADS domain protein integrates floral inductive pathways in Arabidopsis. Genes Dev 14: 2366-2376

Matsushika A, Makino S, Kojima M, Mizuno T (2000) Circadian waves of expression of the APRR1/TOC1 family of PseudoResponse Regulators in Arabidopsis thaliana: Insight into the plant circadian clock. Plant Cell Physiol 41: 1002-1012

Michaels SD, Amasino RM (1999) FLOWERING LOCUS C encodes a novel MADS domain protein that acts as a repressor of flowering. Plant Cell 11: 949-956

Mizoguchi T, Niinuma K, Yoshida R (2007) Day-neutral response of photoperiodic flowering in tomatoes: possible implications based on recent molecular genetics of Arabidopsis and rice. Plant Biotechnol 24: 83-86

Mizoguchi T, Wheatley K, Hanzawa Y, Wright L, Mizoguchi M, Song HR, Carré IA, Coupland G (2002) LHY and CCA1 are partially redundant genes required to maintain circadian rhythms in Arabidopsis. Dev Cell 2: 629-641

Mizoguchi T, Wright L, Fujiwara S, Cremer F, Lee K, Onouchi H, Mouradov A, Fowler S, Kamada H, Putterill J, Coupland G (2005) Distinct roles of GIGANTEA in promoting flowering regulating circadian rhythms in Arabidopsis. Plant Cell 17: 2255-2270

Nakamichi N, Kita M, Ito S, Sato E, Yamashino T, Mizuno T (2005b) The Arabidopsis Pseudo-Response Regulators, PRR5 and PRR9, Coordinately play essential roles for circadian clock function. Plant Cell Physiol 46: 609-619

Nakamichi N, Kita M, Ito S, Yamashino T, Mizuno T (2005a) Pseudo-Response Regulators, $P R R 9, P R R 9, P R R 5$, together play essential roles close to the circadian clock of Arabidopsis thaliana. Plant Cell Physiol 46: 686-698

Nakamichi N, Kita M, Niinuma K, Ito S, Yamashino T, Mizoguchi T, Mizuno T (2007) Arabidopsis clock-associated Pseudo-
Response Regulator PRR9, PRR9 and PRR5 coordinately and Positively regulate Flowering time through the canonical CONSTANS-dependent photoperiodic pathway. Plant Cell Physiol 48: 822-832

Nozue K, Maloof JN (2006) Diurnal regulation of plant growth. Plant Cell Environ 29: 396-408

Park DH, Somers DE, Kim YS, Choy YH, Lim HK, Soh MS, Kim HJ, Kay SA, Nam HG (1999) Control of circadian rhythms photoperiodic flowering by the Arabidopsis GIGANTEA gene. Science 285: 1579-1582

Putterill J, Robson F, Lee K, Simon R, Coupland G (1995) The CONSTANS gene of Arabidopsis promotes flowering and encodes a protein showing similarities to zinc finger transcription factors. Cell 80: 847-857

Quail PH (2002) Phytochrome photosensory signaling networks. Nat Rev Mol Cell Biol 3: 85-93

Roden LC, Song HR, Jackson S, Morris K, Carré IA (2002) Floral responses to photoperiod are correlated with the timing of rhythmic expression relative to dawn dusk in Arabidopsis. Proc Natl Acad Sci 99: 13313-13318

Sawa M, Nusinow DA, Kay SA, Imaizumi T (2007) FKF1 and GIGANTEA complex formation is required for day-length measurement in Arabidopsis. Science 318: 261-265

Schaffer R, Ramsay N, Samach A, Corden S, Putterill J, Carré IA, Coupland G (1998) The late elongated hypocotyl mutation of Arabidopsis disrupts circadian rhythms the photoperiodic control of flowering. Cell 93: 1219-1229

Somers DE, Schultz TF, Milnamow M, Kay SA (2000) ZEITLUPE encodes a novel clock-associated PAS protein from Arabidopsis. Cell 101: 319-329

Somers DE, Webb A, Pearson M, Kay SA (1998) The short-period mutant, toc1-1, alters circadian clock regulation of multiple outputs throughout development in Arabidopsis thaliana. Development 125: 485-494

Staiger D, Apel K, Trepp G (1999) The Atger3 promoter confers circadian clock-regulated transcription with peak expression at the beginning of the night. Plant Mol Biol 40: 873-882

Suarez-Lopez P, Wheatly K, Robson F, Onouchi H, Valverde F, Coupland G (2001) CONSTANS mediates between the circadian clock the control of flowering in Arabidopsis. Nature 410: $1116-1120$

Tamaki S, Matsuo S, Wong HL, Yokoi S, Shinamoto K (2007) Hd3a protein is a mobile flowering signal in rice. Science 316 : 1033-1036

Tsukaya H, Kozuka T, Kim GT (2002) Genetic Control of Petiole Length in Arabidopsis thaliana. Plant Cell Physiol 43: $1221-1228$

Wang ZY, Tobin EM (1998) Constitutive expression of the CIRCADIAN CLOCK ASSOCIATED 1 (CCA1) gene disrupts circadian rhythms suppresses its own expression. Cell 93: 1207-1217

Yamamoto Y, Sato E, Shimizu T, Nakamich N, Sato S, Kato T, Tabata S, Nagatani A, Yamashino T, and Mizuno T (2003) Comparative genetic studies on the APRR5 and APRR9 genes belonging to the APRR1/TOC1 quintet implicated in circadian rhythm, control of flowering time, and early photomorphogenesis. Plant Cell Physiol 44: 1119-1130

Yanovsky MJ, Kay SA (2002) Molecular basis of seasonal time measurement in Arabidopsis. Nature 419: 308-312

Zhong HH, McClung CR (1996) The circadian clock gates expression of two Arabidopsis catalase genes to distinct opposite circadian phases. Mol Gen Genet 251: 196-203 